\title{
Diabetes Risk Perception Among Immigrant and Racial/Ethnic Minority Adults in the U.S.
}

Loretta Hsueh, ${ }^{\mathrm{a}}$ Juan M. Peña, ${ }^{\mathrm{b}}$ Adam T. Hirsh, ${ }^{\mathrm{a}}$ Mary de Groot,,${ }^{\mathrm{c}}$ and Jesse C. Stewart ${ }^{\mathrm{a}}$

${ }^{a}$ Indiana University-Purdue University Indianapolis, Department of Psychology, Indianapolis, IN

${ }^{b}$ University of New Mexico, Department of Psychology, Albuquerque, NM

c Indiana University School of Medicine, Diabetes Translational Research Center, Indianapolis,

IN

Suggested running head: Diabetes risk perception among immigrant and racial/ethnic minority adults

Address Correspondence and Reprint Requests to: Jesse C. Stewart, PhD, Department of Psychology, Indiana University-Purdue University Indianapolis, 402 North Blackford Street, LD100E, Indianapolis, IN 46202. Telephone: (317) 274-6761. Fax: (317) 274-6756. Email: jstew@iupui.edu

This is the author's manuscript of the article published in final edited form as:

Hsueh, L., Peña, J. M., Hirsh, A. T., de Groot, M., \& Stewart, J. C. (2019). Diabetes Risk Perception Among Immigrant and Racial/Ethnic Minority Adults in the United States. The Diabetes Educator, 45(6), 642-651. https://doi.org/10.1177/0145721719873640 


\section{Complete Author Information}

Name: Loretta Hsueh, MA

Correspondence address: 402 North Blackford St., LD119, Indianapolis, IN 46202

Email address: 1hsueh@iu.edu

Name: Juan M. Peña, MS

Correspondence address: 1 University of New Mexico, MSC03-2220, Albuqueque, NM

87131-0001

Email address: penajm@unm.edu

Name: Adam T. Hirsh, PhD

Correspondence address: 402 North Blackford St., LD126M, Indianapolis, IN 46202

Email address: athirsh@iupui.edu

Name: Mary de Groot, PhD

Correspondence address: 410 West $10^{\text {th }}$ St., Suite 3100, Indianapolis, IN 46202

Email address: mdegroot@iu.edu

Name: Jesse C. Stewart, PhD

Correspondence address: 402 North Blackford St., LD100E, Indianapolis, IN 46202

Email address: jstew@iupui.edu 


\section{Compliance with Ethical Standards}

Conflict of Interest: The authors declare that they have no conflict of interest.

Statement of human rights: All procedures performed in studies involving human participants were in accordance with the ethical standards of the institutional and/or national research committee and with the 1964 Helsinki declaration and its later amendments or comparable ethical standards.

Statement on the welfare of animals: This article does not contain any studies with animals performed by any of the authors.

Informed consent: Informed consent was obtained from all individual participants included in the study. 


\begin{abstract}
Purpose: The purpose of the study was to examine associations of immigrant and racial/ethnic status with diabetes risk perception among a population-based sample of U.S. adults without diabetes. Racial/ethnic minorities are at increased risk of developing diabetes. Emerging research shows that immigrant (foreign-born) individuals are also at increased risk, but less is understood about risk perception in this group.
\end{abstract}

Methods: Respondents were 11,569 adults from NHANES 2011-2016 reporting no diabetes or prediabetes. Immigrant status was coded as foreign-born or U.S.-born and used NHANES racial/ethnic categories: White, Black, Mexican American, other Hispanic, Asian, and other/multi-racial. Immigrant status and variables comparing each minority group to Whites were simultaneously entered into models predicting risk perception (yes/no), adjusting for demographic and diabetes risk factors.

Results: Being foreign-born was associated with decreased odds of perceived risk, while being Mexican American, Asian, and other/multi-racial were associated with increased odds of perceived risk.

Discussion: Foreign-born adults are less likely to report perceived risk for diabetes than are U.S.-born adults. Lower diabetes risk perception among immigrants could result in poorer preventative behaviors and later diabetes detection.

Keywords: diabetes; risk perception; emigrants and immigrants; race/ethnicity; health disparities. 


\section{INTRODUCTION}

Public health initiatives urge at-risk individuals to make lifestyle modifications to prevent type 2 diabetes [T2DM] ${ }^{1}$. To increase the potency of such efforts, understanding the role of risk perception is critical ${ }^{2}$. Risk perception refers to an individual's judgment of his or her own likelihood of a negative event. The health belief model ${ }^{3}$ prioritizes risk perception in predicting the likelihood of action and acknowledges the modifying role of sociodemographic factors, such as race/ethnicity.

Although immigrant status has emerged as a social determinant of T2DM health ${ }^{4-6}$, how immigrant status modifies diabetes risk perception remains unknown. This is a critical knowledge gap, as longitudinal evidence suggests immigrants are at greater risk for developing T2DM than their U.S.-born peers ${ }^{5}$. As immigrants and their families contribute substantially to U.S. population growth ${ }^{7}$, promoting preventative health behaviors in this growing population is key to improving overall U.S. health.

While U.S. immigrants are likely to be members of a racial/ethnic minority group, examining diabetes risk perception solely along racial/ethnic lines is insufficient. Several psychosocial and environmental factors may contribute to lower diabetes risk perception among immigrants. Psychosocial factors include familiarity with diabetes risk, which differs by country of origin, and cultural beliefs about the causes of diabetes, which may be stronger among foreign-born individuals compared to co-ethnic U.S.-born peers ${ }^{8-10}$. Environmental factors include a lack of linguistically- and culturally-appropriate public health messaging, potentially contributing to decreased knowledge about diabetes risk factors ${ }^{11,12}$. Examining the unique influence of immigrant status on diabetes risk perception, over and above the influence of race/ethnicity, may facilitate more responsive public health programming. 
No study has examined the association between immigrant status and diabetes risk perception using a population-based sample of adults. Accordingly, the objectives of the present study are to examine associations of (1) immigrant and (2) racial/ethnic status with diabetes risk perception among a population-based sample of U.S. adults without diabetes.

\section{METHODS}

\section{Study Design and Sample}

The institutional review board approved this study. Data was from the National Health and Nutrition Examination Survey (NHANES) 2011-2016 survey years. NHANES employs a cross-sectional, stratified, multistage probability design to capture a nationally representative sample of the civilian, non-institutionalized population. Survey design and procedures are available online (www.cdc.gov/nchs/nhanes.htm). Briefly, about 5,000 individuals were recruited each two-year cycle. Respondents were asked to complete a computer-assisted in-home interview assessing demographic and health-related factors. Approximately 1-2 weeks later, respondents were asked to attend a Mobile Examination Center (MEC) to undergo physical examinations, laboratory assessments, and additional interviews.

All data came from the in-home interviews, except for A1C values and weight and height measurements, which came from the MEC. From the total sample for the 2011-2016 survey years $(N=29,902)$, all respondents aged 18 years or older $(n=17,969)$ who reported having no diabetes/prediabetes diagnosis $(n=14,239)$ were selected. Diabetes diagnosis was determined by asking, "Have you ever been told by a doctor or other health professional that you have diabetes or sugar diabetes?" Respondents answering "No" were included in this sample, and those answering "Yes," "Borderline or Prediabetes," "Refused," "Don’t Know," or did not answer were excluded. Respondents with no diabetes diagnosis were asked about prediabetes, "Have 
you ever been told by a doctor or other health professional that you have any of the following: prediabetes, impaired fasting glucose, impaired glucose tolerance, borderline diabetes or that your blood sugar is higher than normal but not high enough to be called diabetes or sugar diabetes?" Respondents answering "No" were included in this sample. From the 4,239 respondents without self-reported diabetes/prediabetes, respondents with missing data on the outcome, diabetes risk perception ( $n=182)$ were excluded. Respondents with missing data on the predictors country of birth $(n=9)$ and race/ethnicity $(n=0)$ were then excluded. Finally, respondents with missing data on demographic factors $(n=7)$ and diabetes risk-factor covariates, including clinical CVD ( $n=913)$, hypercholesterolemia ( $n=57)$, hypertension $(n=10)$, smoking status ( $n=15)$, family history of diabetes ( $n=223)$, height/weight ( $n=667)$, and A1C $(n=587)$ were excluded. Because this project focuses on the associations between two sociodemographic factors (immigrant status and race/ethnicity) and diabetes risk perception, over and above actual risk, it is important to include these factors as covariates in models predicting diabetes risk perception. The characteristics of the final sample of 11,569 respondents without self-reported diabetes/prediabetes are shown in Table 1.

\section{Measures and Procedures}

Immigrant Status. Respondents indicated country of birth by selecting: "Born in 50 U.S. States or Washington, D.C.," “Other," "Refused," or "Don't Know.” After excluding respondents who answered "Refused," "Don't Know," or did not answer, a dichotomous immigrant status variable coded as U.S.-born ( $0=$ born in U.S. state or Washington, D.C.) or foreign-born (1=born outside of U.S. states or Washington, D.C., including U.S. territories) was computed. 
Race/Ethnicity. Respondents indicated race/ethnicity by selecting: non-Hispanic White, non-Hispanic Black, Mexican American, other Hispanic, non-Hispanic Asian, or other race/ multi-racial. Five dummy variables to represent race/ethnicity were coded, using the nonHispanic White group as the referent.

Diabetes Risk Perception. Diabetes risk perception was assessed by asking, "Do you feel you could be at risk for diabetes or prediabetes?" Participants who answered "Refused," "Don't Know," or did not answer were excluded.

Covariates. Demographic covariates were age (years), sex $(0=$ male, $1=$ female), and four dummy variables for education (referent: college graduate or above). Respondents reported their highest education level: less than $9^{\text {th }}$ grade, $9-11^{\text {th }}$ grade with no diploma, high school diploma or GED, some college or associate degree, and college graduate or above. Diabetes risk factor covariates were: clinical CVD, hypercholesterolemia, hypertension, smoking status, family history of diabetes, body mass index (BMI; $\left.\mathrm{kg} / \mathrm{m}^{2}\right)$, and $\mathrm{A} 1 \mathrm{C}(\%$; a measure of blood glucose control over last 120 days $)^{13}$. Respondents were coded as having clinical CVD ( $0=$ no, $\left.1=y e s\right)$ if they reported a history of coronary artery disease, angina, myocardial infarction, stroke, or congestive heart failure. Variables were computed to represent a history of hypertension $(0=$ no, $1=$ yes $)$, a history of hypercholesterolemia $(0=$ no, $1=$ yes $)$, two dummy variables for smoking status (never versus current smoker, never versus former smoker), and family history of diabetes $(0=$ no, $1=$ yes $)$. Variables for BMI and A1C were continuous.

\section{Data Analysis}

Analyses were performed using SAS version 9.4 (SAS Institute, Cary, NC). T-tests and chi-square $\left(X^{2}\right)$ tests examined differences between foreign-born and U.S.-born respondents, and one-way ANOVAs to examine differences between each racial/ethnic minority group and 
Whites. Appropriate NHANES sampling design weighted variables were applied to all models to account for survey design. Applying sample weights allows each respondent to represent the proportion of the population s/he represents, thus providing estimates representative of the U.S. civilian non-institutionalized population (see www.cdc.gov/nchs/tutorials/nhanes/SurveyDesign/Weighting/intro.htm). Weights from the MEC subsample were used because BMI and A1C were available for this subsample only.

To determine whether immigrant status and race/ethnicity are uniquely associated with diabetes risk perception, two logistic regressions were completed. Immigrant status (foreign-born vs. U.S.-born) and race/ethnicity (five dummy variables with White as referent) were simultaneously into a model with the diabetes risk perception criterion variable (yes/no). Model 1 included demographic factors (age, sex, and four dummy variables for education) as covariates. Model 2 further adjusted for diabetes risk factors (clinical CVD, hypercholesterolemia, hypertension, smoking status, family history of diabetes, BMI, and A1C) to reduce their confounding effect on the associations of interest.

Nonetheless, differences in risk perception could potentially be attributed to differences in objective diabetes risk (i.e., those with higher objective risk are more likely to perceive a risk). To minimize this possibility, a supplemental Model 3 was completed by rerunning Model 2 in the subsample of respondents with clinically-indicated prediabetes (A1C 5.7\%-6.4\%). This subsample was selected because prediabetes represents a critical window for intervention efforts to prevent or delay the development of diabetes. Finally, the interaction between immigrant status and race/ethnicity was tested in a supplementary model using the entire sample. Specifically, five cross-product interaction terms (immigrant status by each of the five 
race/ethnicity dummy variables) were entered into Model 2. In the presence of significant interactions, Model 2 was reanalyzed stratified by race/ethnicity.

\section{RESULTS}

\section{Respondent Characteristics}

In the sample of 11,569 adults without a diabetes/prediabetes diagnosis, approximately one-third (31\%) were foreign-born. As shown in Table 1, foreign-born and U.S.-born respondents differed in several ways. Foreign-born respondents were more likely to be Mexican American, other Hispanic, or Asian and less likely to be White, Black, or other/multi-racial. Foreign-born respondents were more likely be in the lowest (e.g., less than $9^{\text {th }}$ grade) and highest (e.g., college graduate and above) education levels. Foreign-born respondents were less likely to report clinical CVD or hypertension, more likely to have never smoked, less likely to currently smoke, more likely to be a former smoker, and less likely to report a family history of diabetes. Foreign-born respondents also had lower BMI and higher A1C.

Racial/ethnic minority respondents differed from Whites on several demographic factors (see Table 1). For example, minority respondents were more likely to have lower education levels, except for Asians. Asians were more likely to have a college education or above compared to Whites but (along with Mexican Americans and other Hispanics) were also more likely to have received less than a $9^{\text {th }}$ grade education. Similarly, minorities differed from Whites on health-related factors. While Black, Mexican American, and other Hispanic respondents had higher BMIs, Asians had lower BMIs. Further, all minorities had higher A1C, except for other/multi-racial respondents, who did not differ from Whites.

One-quarter $(25 \%)$ of the sample perceived a risk for developing diabetes. Across immigrant status, 769 (21.4\%) foreign-born and 2,135 (26.8\%) U.S.-born respondents reported 
being at risk. Across race/ethnicity, risk perception was highest among other/multi-racial respondents ( $n=126,35.5 \%)$, followed by Mexican American $(n=467,31.2 \%)$, Black $(n=671$, 27.7\%), White ( $n=1055,23.1 \%)$, Asian $(n=321,21.6 \%)$, and other Hispanic $(n=264,21.5 \%)$ respondents. Table 2 displays the frequency of diabetes risk perception according to immigrant status and race/ethnicity.

\section{Associations of Immigrant Status with Diabetes Risk Perception}

Logistic regressions indicated that both immigrant status and race/ethnicity were uniquely associated with diabetes risk perception; however, the nature of the associations differed (Table 3). In Model 1, being foreign-born was associated with 39\% decreased odds of perceiving a diabetes risk $(P<0.001)$, compared to being U.S.-born. Adjusting for diabetes risk factors in Model 2, being foreign-born remained associated with a 27\% decreased odds of diabetes risk perception $(P=0.001)$, compared to being U.S.-born.

\section{Associations of Race/Ethnicity with Diabetes Risk Perception}

In the same two logistic regressions, race/ethnicity was also associated with diabetes risk perception. In Model 1, identifying as a racial/ethnic minority was associated with increased odds of perceiving a diabetes risk (Table 3). In Model 2, being Mexican American, Asian, and other/multi-racial remained associated with increased risk perception (52\% increased odds, $P<0.001 ; 48 \%$ increased odds, $P<0.001 ; 63 \%$ increased odds, $P=0.002$; respectively), whereas being Black and other Hispanic was not $(P=0.056$ and $P=0.702$, respectively).

\section{Associations Among Respondents with Prediabetes}

Among respondents with clinically-indicated prediabetes $(n=3028,26.2 \%$ of the total sample), mean A1C was 5.9\% ( $S D=0.2)$. As is shown in Table 3, being foreign-born remained associated with $35 \%$ decreased odds of perceiving a risk $(P=0.018)$ and being Asian remained 
associated with $89 \%$ increased odds of perceiving a risk $(P=0.014)$. Associations for other racial/ethnic groups were no longer significant $\left(P_{\mathrm{S}}>0.05\right)$ but magnitudes and directions of the associations were similar to associations in the total sample.

\section{Race/Ethnicity as a Moderator of the Immigrant Status-Diabetes Risk Perception Association}

The moderating effect of race/ethnicity on the immigrant status-diabetes risk perception association was examined by entering five interactions terms (immigrant status by each of the five dummy-coded race/ethnicity variables) into Model 2. The three interactions of immigrant status by Black, Mexican American, and other/multi-racial (compared to White) were significant $(P \mathrm{~s}<0.01)$, whereas the others were not $\left(P_{s}>0.05\right)$. To probe these interaction terms, analyses were stratified by race/ethnicity (Figure 1). For Black, Mexican American, other Hispanic, and other/multi-racial groups, immigrant status was not associated with risk perception $\left(P_{\mathrm{S}}>0.10\right)$. Immigrant status was associated with decreased odds of risk perception among Whites and Asians $(P=0.002$ and $P=0.005$, respectively). Notably, results of stratified analyses were consistent with the raw rates reported in Table 2.

\section{DISCUSSION}

The aim of this study was to examine the unique associations between immigrant and racial/ethnic status and diabetes risk perception. In a large, nationally representative sample of U.S. adults without diabetes or prediabetes, being foreign-born was associated with lower odds of perceived diabetes risk, over and above the effect of race/ethnicity. Immigrant status' negative association with diabetes risk perception persisted after adjustment for potential confounders, including demographic and diabetes risk factors. Further, race/ethnicity showed a positive association with risk perception for Mexican American, Asian, and other/multi-racial groups. 
After minimizing group differences in actual diabetes risk by exploring these associations among the subsample of respondents with prediabetes, the magnitude and direction of associations remained similar to those observed in the total sample. These results, combined with Model 2 results adjusting for diabetes risk factors in the entire sample, suggest that differences in actual diabetes risk are unlikely to account for the observed group differences in perceived risk. Finally, exploratory analyses suggest that foreign-born White and Asian groups may be driving the overall immigrant status effect, with foreign-born White and Asian adults having decreased odds of perceiving a diabetes risk compared to U.S.-born peers of the same ethnicity.

Together, these findings suggest that U.S. immigrants perceive themselves to be at lower risk for developing diabetes, despite emerging empirical evidence to the contrary ${ }^{5}$. Mismatch between risk perception and actual risk may have important consequences, as lower risk perception decreases the likelihood of engaging in lifestyle modifications to prevent or delay diabetes onset ${ }^{3}$. Further, findings indicate that immigrant and racial/ethnic status are uniquely associated with different diabetes risk perceptions, with immigrants less likely, but racial/ethnic minorities generally more likely, to perceive a diabetes risk. Using the evidence base for risk perception based on race/ethnicity (which is more developed) to assess risk perception among immigrants may hinder effective public health programming for immigrants.

Although not the primary objective, findings on diabetes risk perception by race/ethnicity coalesce with existing evidence. In prior studies of people without diabetes, racial/ethnic minorities were more likely to perceive diabetes risk compared to non-Hispanic Whites. These sampled Filipino, Chinese, and Latinos living in California ${ }^{14}$, undergraduates in Texas and Arizona ${ }^{15,16}$, and women in Appalachia ${ }^{15}$. In addition, a 2009 study of older (45+) adults without diabetes found that being Native American, Alaska Native, Asian American, Pacific 
Islander, or Native Hawaiian was associated with higher perceived risk; however, this association was not found for African Americans or Hispanics/Latinos ${ }^{17}$. The current findings add to this by confirming some of these racial/ethnic differences using a large, nationally representative sample of adults, including younger adults. Further, Yang et al. ${ }^{18}$ used NHANES data to examine specific reasons for perceiving diabetes risk versus the presence of actual risk factors. They found that Asians were less likely to correctly perceive weight (compared to Whites) and low physical activity (compared to Blacks, Mexican Americans, and other Hispanics) as diabetes risk factors. The present study contextualizes Yang et al.'s findings by showing that perceived diabetes risk does indeed differ by race/ethnicity. Together, these results suggest that although Asians may perceive a higher risk for developing diabetes, they may be less aware of specific risk factors. As a result, Asians may fail to address all of the relevant aspects of preventing or delaying diabetes.

Although this is the first study to examine the association of immigrant status with diabetes risk perception, findings are consistent with other studies implicating immigrant status as a social determinant of diabetes health. As previously described, Oza-Frank et al. ${ }^{5}$ found that foreign-born adults were at greater risk for incident diabetes than U.S.-born adults. Crosssectional results from two nationally representative databases suggests immigrants diagnosed with diabetes are less likely to receive, or engage in, optimal disease management. In the Medical Expenditure Panel Survey, immigrants were less likely to have met at least one of seven ADA-recommended diabetes self-management benchmarks ${ }^{19}$. Results from this research group's recent NHANES study found that, among adults with a diabetes diagnosis, being foreign-born was associated with decreased odds of receiving insulin treatment, compared to being U.S.-born ${ }^{6}$. The present findings add to this by highlighting diabetes risk perception as a 
potential intervention target. However, research examining the antecedents and consequences of lower perceived risk is warranted, as unexamined factors may modify the effect of risk perception on the likelihood of engaging in lifestyle modifications for immigrant communities specifically. For example, other modifiers in the health belief model include cues to action (e.g., symptom recognition) and media information (e.g., public health programming).

Several factors may explain the lower risk perception among immigrants. A lack of linguistically- and culturally-appropriate public health messaging likely contributes to decreased diabetes risk knowledge ${ }^{11,12}$. If known risk factors (such as high BMI, smoking, and a positive family history of diabetes) are not as strongly associated with diabetes for immigrants as they are for the general population, then these weakened associations may be a mechanism through which immigrant status becomes associated with lower risk perception. Resnicow et al. ${ }^{20}$ argue that targeting aspects of observable culture, such as language, are important for increasing the acceptability of health-related messages; however, achieving message saliency requires incorporating cultural, social, historical, environmental and psychological realities that motivate the target health behavior. For U.S. immigrants, this may mean acknowledging, and providing methods for overcoming, the limitations immigrants face in achieving health, including lower insurance coverage rates and familiarity with an increasingly complex healthcare system ${ }^{21-24}$. Still, having requisite diabetes risk knowledge may be insufficient. Walker et al. ${ }^{25}$ surveyed adults with diabetes on their risk perceptions for diabetes-related complications and found that foreign-born adults were more susceptible to optimistic bias, meaning they perceived themselves to be at less risk for complications compared to others of the same age/gender. Optimistic bias may also contribute to lower diabetes risk perception among those who have not yet developed diabetes, but no studies of this currently exist. Outside of the diabetes context, evidence suggests 
that U.S. immigrants exhibit strong optimistic beliefs about the future for themselves and their families ${ }^{26-29}$. The degree to which this 'immigrant optimism' ${ }^{27}$ applies to health risk perceptions is an avenue for future research.

Among the study's strengths is the use of a large, nationally representative sample of adults without a diabetes/prediabetes diagnosis and data collection through interviews, physical examinations, and laboratory tests. In particular, the physical and laboratory components provided objective assessments of BMI and A1C. Some limitations are worth noting. First, although models were adjusted for potential confounders including actual diabetes risk factors, individuals could not be matched on these factors. Rates of certain diabetes risk factors (e.g., CVD) were lower among foreign-born adults; however, most were assessed via self-report, and it is possible that immigrants were less likely to have received these diagnoses. Importantly, foreign-born adults had higher A1C, as objectively measured at the MEC, and associations persisted in this subsample of adults with prediabetes. Second, respondents who self-reported no history of physician-diagnosed diabetes were selected. Because immigrants may be more likely to have undiagnosed diabetes ${ }^{30}$, this sample may have included undiagnosed diabetes cases, especially among foreign-born adults, thus underestimating their low levels of risk perception observed herein.

Several implications for research and practice are worth considering. In terms of research, the finding that immigrant and racial/ethnic status are each associated with opposing diabetes risk perception outcomes should motivate future research examining why and how these differences arise. This is particularly intriguing when considering the unequal distribution of foreign-born and U.S.-born individuals within certain racial/ethnic groups-i.e., more foreignborn adults in the Asian group and more U.S.-born adults are in the White and Black groups. 
While exploratory analyses stratified by race/ethnicity suggest the immigrant status-risk perception association is driven by Whites and Asians, low cell counts of foreign-born (among White) and U.S.-born (among Asian) individuals endorsing diabetes risk perception constrains confidence in these interpretations. Nonetheless, these tentative findings should encourage future research to examine and compare rates (and reasons) of immigrants' diabetes risk perception across race/ethnicity. With respect to practice, public health campaigns to increase diabetes risk perception in high-risk groups should consider developing programs specifically for foreign-born populations. Such immigrant-focused interventions have been in place for smoking cessation and nutritional education ${ }^{31,32}$.

\section{NEW CONTRIBUTION TO THE LITERATURE}

Foreign-born adults are less likely to report perceived risk for diabetes than are U.S. -born adults. Lower diabetes risk perception among immigrants could result in poorer preventative behaviors and later detection, thus contributing to immigrant health disparities in diabetes. 
Acknowledgements: Data were presented at the 2017 Annual Meeting and Scientific Sessions of the Society of Behavioral Medicine in San Diego, CA.

Funding: No financial or material support was used in this study.

Declaration of Interest: The authors declare no conflicts of interest. 


\section{REFERENCES}

1. Green LW, Brancati FL, Albright A, Group PPoDW. Primary prevention of type 2 diabetes: integrative public health and primary care opportunities, challenges and strategies. Fam Pract. 2012;29(suppl_1):i13-i23.

2. Ferrer RA, Klein WM. Risk perceptions and health behavior. Curr Opin Psychol. $2015 ; 5: 85-89$.

3. Janz NK, Becker MH. The health belief model: A decade later. Health Educ $Q$. $1984 ; 11(1): 1-47$.

4. Hill JO, Galloway JM, Goley A, et al. Scientific statement: socioecological determinants of prediabetes and type 2 diabetes. Diabetes Care. 2013;36(8):2430-2439.

5. Oza-Frank R, Chan C, Liu K, Burke G, Kanaya AM. Incidence of Type 2 Diabetes by Place of Birth in the Multi-Ethnic Study of Atherosclerosis (MESA). J Immigr Minor Health. 2013;15(5):918-924.

6. Hsueh L, Vrany EA, Patel JS, et al. Associations between immigrant status and pharmacological treatments for diabetes in US adults. Health Psychol. 2018;37(1):61-69.

7. López G, Bialik K. Key findings about US immigrants. Pew Research Center. 2017;3.

8. Landrine H, Klonoff EA. Culture and health-related schemas: A review and proposal for interdisciplinary integration. Health Psychol. 1992;11(4):267.

9. Caban A, Walker EA. A systematic review of research on culturally relevant issues for Hispanics with diabetes. Diabetes Educ. 2006;32(4):584-595.

10. Guariguata L, Whiting DR, Hambleton I, Beagley J, Linnenkamp U, Shaw JE. Global estimates of diabetes prevalence for 2013 and projections for 2035. Diabetes Res Clin Pract. 2014;103(2):137-149. 
11. Clayman ML, Manganello JA, Viswanath K, Hesse BW, Arora NK. Providing health messages to Hispanics/Latinos: understanding the importance of language, trust in health information sources, and media use. J Health Commun. 2010;15(sup3):252-263.

12. Maty S, Tippens K. Perceived and actual diabetes risk in the Chinese and Hispanic/Latino communities in Portland, OR, USA. Diabetic Med. 2011;28(6):658-667.

13. Saudek CD, Derr RL, Kalyani RR. Assessing glycemia in diabetes using self-monitoring blood glucose and hemoglobin A(1c). JAMA. 2006;295(14):1688-1697.

14. Fukuoka Y, Choi J, Bender MS, Gonzalez P, Arai S. Family history and body mass index predict perceived risks of diabetes and heart attack among community-dwelling Caucasian, Filipino, Korean, and Latino Americans-DiLH Survey. Diabetes Res Clin Pract. 2015;109(1):157-163.

15. Chopra I, Chopra A. Risk perception for diabetes in Appalachian women. Women Health. 2017;57(5):534-550.

16. Dickerson JB, Smith ML, Sosa E, McKyer EL, Ory MG. Perceived risk of developing diabetes in early adulthood: beliefs about inherited and behavioral risk factors across the life course. J Health Psychol. 2012;17(2):285-296.

17. Gallivan J, Brown CS, Greenberg R, Clark CM. Predictors of perceived risk of the development of diabetes. Diabetes Spectr. 2009;22(3):163-169.

18. Yang K, Baniak LM, Imes CC, Choi J, Chasens ER. Perceived Versus Actual Risk of Type 2 Diabetes by Race and Ethnicity. Diabetes Educ. 2018:0145721718770983.

19. Dallo FJ, Wilson FA, Stimpson JP. Quality of diabetes care for immigrants in the US. Diabetes Care. 2009;32(8):1459-1463. 
20. Resnicow K, Baranowski T, Ahluwalia JS, Braithwaite RL. Cultural sensitivity in public health: defined and demystified. Ethn Dis. 1999;9(1):10-21.

21. Siddiqi A, Zuberi D, Nguyen QC. The role of health insurance in explaining immigrant versus non-immigrant disparities in access to health care: Comparing the United States to Canada. Soc Sci Med. 2009;69(10):1452-1459.

22. Ku L, Matani S. Left out: immigrants' access to health care and insurance. Health Aff (Millwood). 2001;20(1):247-256.

23. Son E, Moring NS, Igdalsky L, Parish SL. Navigating the health-care system in community: Perspectives from Asian immigrant parents of children with special healthcare needs. J Child Health Care. 2018:1367493517753084.

24. Chi JT, Handcock MS. Identifying Sources of Health Care Underutilization Among California’s Immigrants. J Racial Ethn Health Disparities. 2014;1(3):207-218.

25. Walker EA, Caban A, Schechter CB, et al. Measuring comparative risk perceptions in an urban minority population. Diabetes Educ. 2007;33(1):103-110.

26. Viruell-Fuentes EA. Beyond acculturation: immigration, discrimination, and health research among Mexicans in the United States. Soc Sci Med. 2007;65(7):1524-1535.

27. Kao G, Tienda M. Optimism and achievement: The educational performance of immigrant youth. Soc Sci Q. 1995:1-19.

28. Suárez-Orozco C, Suárez-Orozco MM. Children of immigration. Harvard University Press; 2009.

29. Lee H-S, Brown SL, Mitchell MM, Schiraldi GR. Correlates of resilience in the face of adversity for Korean women immigrating to the US. J Immigr Minor Health. $2008 ; 10(5): 415-422$. 
30. Barcellos SH, Goldman DP, Smith JP. Undiagnosed Disease, Especially Diabetes, Casts Doubt On Some Of Reported Health 'Advantage' Of Recent Mexican Immigrants. Health Aff (Millwood). 2012;31(12):2727-2737.

31. Wetter DW, Mazas C, Daza P, et al. Reaching and treating Spanish-speaking smokers through the National Cancer Institute's cancer information service. Cancer. 2007;109(S2):406-413.

32. Elder JP, Candelaria JI, Woodruff SI, Criqui MH, Talavera GA, Rupp JW. Results of language for health: cardiovascular disease nutrition education for Latino English-as-asecond-language students. Health Educ Behav. 2000;27(1):50-63. 
Table 1. Differences in Respondent Characteristics by Immigrant Status and Race/Ethnicity in NHANES 2011-2016

\begin{tabular}{|c|c|c|c|c|c|c|c|c|c|}
\hline & $\begin{array}{c}\text { Total } \\
\text { Sample } \\
(n=11569)\end{array}$ & $\begin{array}{l}\text { U.S.-born } \\
(n=7971)\end{array}$ & $\begin{array}{c}\text { Foreign-born } \\
\quad(n=3598)\end{array}$ & $\begin{array}{c}\text { Non- } \\
\text { Hispanic } \\
\text { White } \\
(n=4576)\end{array}$ & $\begin{array}{c}\text { Non- } \\
\text { Hispanic } \\
\text { Black } \\
(n=\mathbf{2 4 2 3}) \\
\end{array}$ & $\begin{array}{l}\text { Mexican } \\
\text { American } \\
(n=1498)\end{array}$ & $\begin{array}{c}\text { Other } \\
\text { Hispanic } \\
(n=1228)\end{array}$ & $\begin{array}{c}\text { Non- } \\
\text { Hispanic } \\
\text { Asian } \\
(n=1489)\end{array}$ & $\begin{array}{c}\text { Other/Multi- } \\
\text { racial } \\
(n=355)\end{array}$ \\
\hline \multicolumn{10}{|l|}{ Demographic Factors } \\
\hline Age, Years & $46.1(17.4)$ & $46.2(18.2)$ & $46.0(15.4)$ & $49.2(18.7)$ & $44.9(16.6)^{\dagger \dagger}$ & $43.4(15.8)^{\dagger \dagger}$ & $46.2(16.5)^{\dagger \dagger}$ & $43.3(15)^{\dagger \dagger}$ & $39.6(15.8)^{\dagger \dagger}$ \\
\hline Female, \% & $6000(51.9)$ & $4143(52.0)$ & $1857(51.6)$ & $2345(51.2)$ & $1276(52.7)$ & $785(52.4)$ & $679(55.3)^{\dagger}$ & $752(50.5)$ & $163(45.9)$ \\
\hline Immigrant, \% & $3598(31.1)$ & - & - & $204(4.5)$ & $298(12.3)$ & $875(58.4)^{\dagger \dagger}$ & $871(70.9)^{\dagger \dagger}$ & $1281(86.0)^{\dagger \dagger}$ & $69(19.4)^{\dagger \dagger}$ \\
\hline \multicolumn{10}{|l|}{ Education, \% } \\
\hline Less Than 9th Grade & $935(8.1)$ & $241(3.0)$ & $694(19.3)^{* *}$ & $132(2.9)$ & $81(3.3)$ & $407(27.2)^{\dagger \dagger}$ & $207(16.9)^{\dagger \dagger}$ & $101(6.8)^{\dagger \dagger}$ & $7(2.0)$ \\
\hline $\begin{array}{l}\text { 9-11th Grade } \\
\text { High School }\end{array}$ & $1449(12.5)$ & $961(12.1)$ & $488(13.6)^{*}$ & $461(10.1)$ & $368(15.2)^{\dagger \dagger}$ & $316(21.1)^{\dagger \dagger}$ & $175(14.3)^{\dagger \dagger}$ & $97(6.5)^{\dagger \dagger}$ & $32(9.0)$ \\
\hline $\begin{array}{l}\text { Graduate/GED } \\
\text { Some College or AA }\end{array}$ & 2495 (21.6) & $1869(23.4)$ & $626(17.4)^{* *}$ & $987(21.6)$ & $656(27.1)^{\dagger \dagger}$ & $300(20.0)$ & $274(22.3)$ & $203(13.6)^{\dagger \dagger}$ & $75(21.1)$ \\
\hline Degree & $3569(30.8)$ & $2814(35.3)$ & $755(21.0)^{* *}$ & $1556(34.0)$ & $868(35.8)$ & $341(22.8)^{\dagger \dagger}$ & $354(28.8)^{\dagger \dagger}$ & $303(20.3)^{\dagger \dagger}$ & $147(41.4)^{\dagger}$ \\
\hline College Graduate or Above & $3121(27.0)$ & $2086(26.2)$ & $1035(28.8)^{*}$ & $1440(31.5)$ & $450(18.6)^{\dagger \dagger}$ & $134(8.9)^{\dagger \dagger}$ & $218(17.8)^{\dagger \dagger}$ & $785(52.7)^{\dagger \dagger}$ & $94(26.5)^{\dagger}$ \\
\hline \multicolumn{10}{|l|}{ Diabetes Risk Factors } \\
\hline Cardiovascular Disease, \% & $812(7.0)$ & $667(8.4)$ & $145(4.0)^{* *}$ & $442(9.7)$ & $173(7.1)^{\dagger \dagger}$ & $58(3.9)^{\dagger \dagger}$ & $69(5.6)^{\dagger \dagger}$ & $39(2.6)^{\dagger \dagger}$ & $31(8.7)$ \\
\hline Hypercholesterolemia, \% & $3179(27.5)$ & $2187(27.4)$ & $992(27.6)$ & $1439(31.4)$ & $586(24.2)^{\dagger \dagger}$ & $347(23.2)^{\dagger \dagger}$ & $369(30.0)$ & $355(23.8)^{\dagger \dagger}$ & $83(23.4)^{\dagger}$ \\
\hline Hypertension, \% & $3289(28.4)$ & $2478(31.1)$ & $811(22.5)^{* *}$ & $1412(30.9)$ & $878(36.2)^{\dagger \dagger}$ & $325(21.7)^{\dagger \dagger}$ & $328(26.7)^{\dagger \dagger}$ & $258(17.3)^{\dagger \dagger}$ & $88(24.8)^{\dagger}$ \\
\hline \multicolumn{10}{|l|}{ Smoking Status, \% } \\
\hline Never & $6808(58.5)$ & $4259(53.4)$ & $2549(70.8)^{* *}$ & $2289(50.0)$ & $1446(59.7)^{\dagger \dagger}$ & $1002(66.9)^{\dagger \dagger}$ & $760(61.9)^{\dagger \dagger}$ & $1131(76.0)^{\dagger \dagger}$ & $180(50.7)$ \\
\hline Current & $2361(20.4)$ & $1947(24.4)$ & $414(11.5)^{* *}$ & $1087(23.8)$ & $611(25.2)^{\dagger \dagger}$ & $212(14.2)^{\dagger \dagger}$ & $191(15.6)^{\dagger}$ & $149(10.0)^{\dagger \dagger}$ & $111(31.3)^{\dagger \dagger}$ \\
\hline Former & $2400(20.7)$ & $1765(22.1)$ & $635(17.6)^{* *}$ & $1200(26.2)$ & $366(15.1)$ & $284(19.0)^{\dagger \dagger}$ & $277(22.6)^{\dagger \dagger}$ & $209(14.0)^{\dagger \dagger}$ & $64(18.0)^{\dagger}$ \\
\hline Family History of Diabetes, \% & $4130(35.7)$ & $2968(37.2)$ & $1162(32.3)^{* *}$ & $1436(31.4)$ & $1050(43.3)^{\dagger \dagger}$ & $635(42.4)^{\dagger \dagger}$ & $398(32.4)$ & $476(32.0)$ & $135(38.0)^{\dagger}$ \\
\hline Body Mass Index, $\mathrm{kg} / \mathrm{m}^{2}$ & $28.4(6.7)$ & $29(7.1)$ & $27.1(5.3) * *$ & $28.2(6.6)$ & $30(7.7)^{\dagger \dagger}$ & $30(6.4)^{\dagger \dagger}$ & $28.8(6.0)^{\dagger}$ & $24.4(4.0)^{\dagger \dagger}$ & $28.4(6.8)$ \\
\hline Hemoglobin A1c Level, \% & $5.5(0.6)$ & $5.4(0.5)$ & $5.5(0.6) * *$ & $5.4(0.5)$ & $5.6(0.6)^{\dagger \dagger}$ & $5.5(0.6)^{\dagger \dagger}$ & $5.5(0.6)^{\dagger \dagger}$ & $5.5(0.6)^{\dagger \dagger}$ & $5.4(0.6)$ \\
\hline
\end{tabular}

Note: Continuous variables presented as mean and $S D$, categorical variables presented as $n$ and \%. NHANES, National Health and Nutrition Examination Survey; U.S., United States; GED, General Education Diploma; AA, Associate degree.

*Significant difference between U.S.-born and foreign-born, $\mathrm{p}<0.05$

** Significant difference between U.S.-born and foreign-born, $\mathrm{p}<0.001$

$\uparrow$ Significant difference between racial/ethnic minority group and non-Hispanic white group, $p<0.05$

iं Significant difference between racial/ethnic minority group and non-Hispanic white group, $\mathrm{p}<0.001$ 
Table 2. Frequency of Diabetes Risk Perception by Immigrant Status and Race/Ethnicity, NHANES 2011-2016

\begin{tabular}{lccc}
\hline & $\begin{array}{c}\text { U.S.-born } \\
\mathbf{n}(\%)\end{array}$ & $\begin{array}{c}\text { Foreign-born } \\
\mathbf{n}(\%)\end{array}$ & $\begin{array}{c}\text { Total } \\
\mathbf{n}(\boldsymbol{\%})\end{array}$ \\
\hline Non-Hispanic White & $1026(23.5 \%)$ & $29(14.2 \%)$ & $1055(23.1 \%)$ \\
Non-Hispanic Black & $606(28.5 \%)$ & $65(21.8 \%)$ & $671(27.7 \%)$ \\
Mexican American & $233(37.4 \%)$ & $234(26.7 \%)$ & $467(31.2 \%)$ \\
Other Hispanic & $105(29.4 \%)$ & $159(18.3 \%)$ & $264(21.5 \%)$ \\
Non-Hispanic Asian & $61(29.3 \%)$ & $260(20.3 \%)$ & $321(21.6 \%)$ \\
Other/Multi-racial & $104(36.4 \%)$ & $22(31.9 \%)$ & $126(35.5 \%)$ \\
Total & $2135(26.8 \%)$ & $769(21.4 \%)$ & $2904(25.1 \%)$ \\
\hline
\end{tabular}

NHANES, National Health and Nutrition Examination Survey; U.S., United States. 
Table 3. Odds Ratios and Confidence Intervals for Logistic Regression Models Examining Associations of Immigrant Status and Race/Ethnicity with Risk Perception, NHANES 2011-2016

\begin{tabular}{|c|c|c|c|}
\hline & Model 1: Demographic Factors ${ }^{\dagger}$ & $\begin{array}{l}\text { Model 2: Demographic and } \\
\text { Diabetes Risk Factors }^{\dagger}\end{array}$ & $\begin{array}{l}\text { Model 3: Demographic and } \\
\text { Diabetes Risk Factors among } \\
\text { Subsample with Prediabetes }{ }^{\dagger \dagger}\end{array}$ \\
\hline & OR $(95 \% C I)$ & OR $(95 \% C I)$ & OR $(95 \% C I)$ \\
\hline \multicolumn{4}{|l|}{ Immigrant Status } \\
\hline U.S.-Born & Ref. & Ref. & Ref. \\
\hline Foreign-Born & $0.613^{* *}(0.519-0.724)$ & $0.732 * *(0.610-0.878)$ & $0.649 *(0.454-0.928)$ \\
\hline \multicolumn{4}{|l|}{ Race/Ethnicity } \\
\hline Non-Hispanic White & Ref. & Ref. & Ref. \\
\hline Non-Hispanic Black & $1.261 *(1.086-1.456)$ & $0.855(0.728-1.004)$ & $0.850(0.610-1.185)$ \\
\hline Mexican American & $1.997 * *(1.709-2.335)$ & $1.520 * *(1.242-1.861)$ & $1.569(0.997-2.471)$ \\
\hline Other Hispanic & $1.225^{*}(1.009-1.489)$ & $1.047(0.828-1.323)$ & $1.027(0.632-1.666)$ \\
\hline Non-Hispanic Asian & $1.389 *(1.137-1.696)$ & $1.475^{* *}(1.184-1.837)$ & $1.889 *(1.138-3.134)$ \\
\hline Other/Multi-Racial & $1.828 * *(1.436-2.328)$ & $1.634 *(1.202-2.223)$ & $1.082(0.512-2.286)$ \\
\hline
\end{tabular}

NHANES, National Health and Nutrition Examination Survey; Ref., reference group; U.S., United States.

${ }^{\dagger} n=11569,{ }^{\dagger} n=3028$

$* p<0.05$

$* * p<0.001$ 


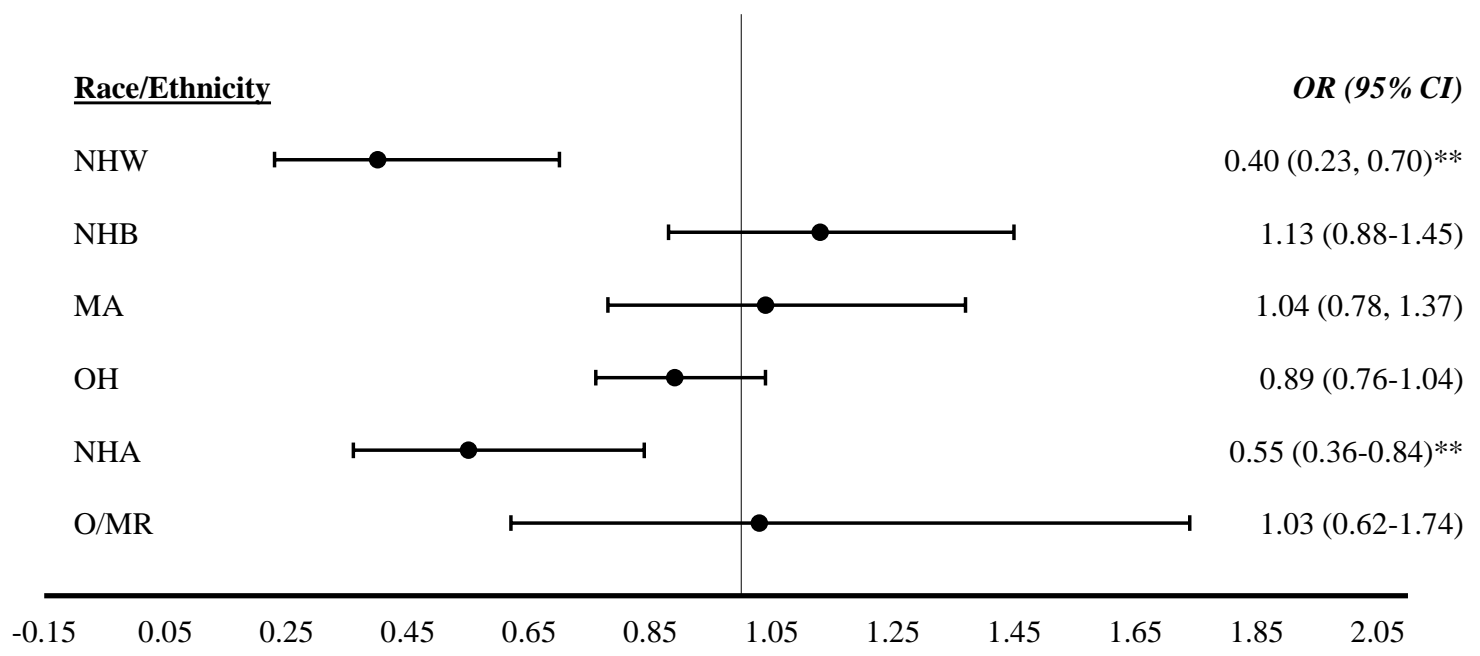

Figure 1. Forest plot summarizing odds ratio estimates of association of being foreignborn with diabetes risk perception, stratified by six race/ethnicity groups. Data from NHANES 2011-2016. Models adjusted for age, sex, education, clinical cardiovascular disease, hypercholesterolemia, hypertension, smoking status, family history of diabetes, body mass index, hemoglobin A1c level, and NHANES sampling design. NHW = nonHispanic White; NHB = non-Hispanic Black; MA = Mexican American; $\mathrm{OH}=$ other Hispanic; NHA = non-Hispanic Asian; O/MR = other/multi-racial; NHANES = National Health and Nutrition Examination Survey. 